\title{
ROUTING AND WAVELENGTH ASSIGNMENT FOR SCHEDULED AND RANDOM LIGHTPATH DEMANDS: BIFURCATED ROUTING VERSUS NON-BIFURCATED ROUTING
}

\author{
Mohamed KOUBAA, Nicolas PUECH, and Maurice GAGNAIRE \\ Telecom Paris - LTCI - UMR 514I CNRS, Paris, France
}

\begin{abstract}
We consider the routing and the wavelength assignment (RWA) of scheduled and random lightpath demands in a wavelength switching mesh network without wavelength conversion functionality. Scheduled lightpath demands (SLDs) are connection demands for which the set-up and tear-down times are known in advance as opposed to random lightpath demands (RLDs) which are dynamically established and released according to a random pattern of requests. Two routing strategies are proposed which process the SLDs and the RLDs separately. The first routing strategy allows to bifurcate the traffic on several routes connecting the source to the destination of a demand whereas the second strategy forces atomic routing. The routing strategies are compared through rejection ratio.
\end{abstract}

\section{INTRODUCTION}

Routing and wavelength assignment in optical transport networks has been extensively investigated for planning and traffic engineering purposes. RWA problems are often classified according to the nature of the considered traffic demands, namely, whether they are static or dynamic [1]. In the static RWA problem where the requests are known in advance, the problem is to set-up lightpaths while minimizing network resource usage such as the number of requested WDM channels or the number of requested wavelengths $[2,3]$. The set of established lightpaths remain in the network for a long period of time. Dynamic RWA deals with connections that arrive dynamically (randomly) [4]. The performance of dynamic RWA is often measured through some network performance metric (e.g., blocking probability also called rejection ratio) $[5,6]$. 
In this paper, we deal with the routing and wavelength assignment (RWA) problem in WDM all-optical transport networks. We consider two types of traffic demands: scheduled demands and random demands. Static demands are not considered here since, once established, these demands remain in the network for a long time. This can be seen as a reduction in the number of available wavelengths on some network links. A scheduled lightpath demand (SLD) [7] is a connection demand represented by a tuple $(s, d, n, \alpha, \omega)$, where $s$ and $d$ are the source and destination nodes of the demand, $n$ is the number of requested lightpaths, and $\alpha, \omega$ are respectively the set-up and tear-down dates of the demand. The SLD model is deterministic because the demands are known in advance and is dynamic because it takes into account the evolution of the traffic load in the network over time. A random lightpath demand (RLD) corresponds to a connection request that arrives randomly and is dealt with on the fly. We use the same tuple notation to describe an RLD. To the best of our knowledge, this is the first time that both deterministic and dynamic traffic demands are considered simultaneously to address the routing and wavelength assignment problem in WDM all-optical transport networks.

The routing strategies studied in this paper aim at establishing random lightpath demands on the fly, provided that the RWA for the scheduled lightpath demands has already been calculated. The first routing strategy allows $b i$ furcated routing: the requested lightpaths of any demand may follow distinct paths from the source to the destination of the demand. Conversely, the second routing strategy imposes atomic routing also called non-bifurcated routing: all the requested lightpaths of a demand have to be routed on the same path. We compute the rejection ratio and discuss the advantages for each strategy by measuring the improvement of the rejection ratio via bifurcated routing.

We call a span the physical pipe connecting two adjacent nodes $u$ and $v$ in the network. Fibers laid down in a span may have opposite directions. We assume here that a span $(u, v)$ is made of two opposite unidirectional fibers. As opposed to a span, a link or a fiber-link refers to a single unidirectional fiber connecting node $u$ to node $v$. The bandwidth of each optical fiber is wavelength-division demultiplexed into a set of $\chi$ wavelengths, $\Lambda=\left\{\lambda_{1}, \lambda_{2}, \ldots, \lambda_{\chi}\right\}$. When a wavelength is used by a lightpath, it is busy (in one direction). A lightpath connecting a node $s$ to a node $d$ is defined by a physical route in the network (a path) connecting $s$ to $d$ and a wavelength $\lambda$ such that $\lambda$ is available on every fiber-link of this route. A path-free wavelength is a wavelength which is not used by any lightpath on any fiber-link of the considered path. A lightpath demand is rejected (blocked) when there are not enough available network resources to satisfy it. The rejection ratio $(R r)$ is the ratio of the number of rejected demands to the total number of lightpath demands arrived at the network. 
The remainder of the paper is organized as follows. In Section 2, we describe the mathematical model and the algorithms of the studied RWA strategies. We then (Section 3) propose some simulation results obtained with them and compare our strategies in terms of rejection ratio. Finally, in Section 4, we draw some conclusions and set directions for future work.

\section{THE STUDIED RWA ALGORITHMS}

In this section, we describe the algorithms used for the routing and wavelength assignment of SLDs and RLDs. Both algorithms deal with the SLDs and the RLDs in two separate phases. The first phase computes the RWA for the SLDs and aims at minimizing the number of blocked SLDs. Taking the assignment of the SLDs into account, the second phase computes the RWA for the RLDs.

\subsection{Mathematical model}

Here are the notations used to describe a lightpath demand (LD), be it scheduled or random.

- $G=(V, E, \vartheta)$ is an arc-weighted symmetrical directed graph with vertex set $V=\left\{v_{1}, v_{2}, \ldots, v_{N}\right\}$, arc set $E=\left\{e_{1}, e_{2}, \ldots, e_{L}\right\}$ and weight function $\vartheta: E \rightarrow \mathbb{R}_{+}$mapping the physical length (or any other cost of the links set by the network operator for example).

- $N=|V|, L=|E|$ are respectively the number of nodes and links in the network.

- $D$ denotes the total number of SLDs and RLDs arrived at the network during the observation period.

- The LD number $i, 1 \leq i \leq D$, to be established is defined by a tuple $\left(s_{i}, d_{i}, n_{i}, \alpha_{i}, \omega_{i}\right) . s_{i} \in V, d_{i} \in V$ are the source and the destination nodes of the demand, $n_{i}$ is the number of requested lightpaths, and $\alpha_{i}$ and $\omega_{i}$ are respectively the set-up and tear-down dates of the demand.

- $P_{k, i}, 1 \leq k \leq K, 1 \leq i \leq D$, represents the $k^{t h}$ alternate shortest path in $G$ connecting node $s_{i}$ to node $d_{i}$ (source and destination of the $i^{t h}$ demand). We compute $K$ alternate shortest paths for each sourcedestination pair according to the algorithm described in [9] (if so many paths exist, otherwise we only consider the available ones).

- $\kappa_{k, i, t}=\left(\gamma_{1, k}^{i, t}, \gamma_{2, k}^{i, t}, \ldots, \gamma_{\chi, k}^{i, t}\right)$ is a $\chi$-dimensional binary vector. $\gamma_{j, k}^{i, t}=$ $1,1 \leq j \leq \chi$, if $\lambda_{j}$ is a path-free wavelength along the path $P_{k, i}$, from $s_{i}$ to $d_{i}$ at time $t$. Otherwise $\gamma_{j, k}^{i, t}=0$.

- $\sigma_{k, i, t}=\sum_{j=1}^{\chi} \gamma_{j, k}^{i, t}$ is the number of path-free wavelengths along $P_{k, i}$ at time $t$. 
$\delta$ will denote an SLD whereas $\tau$ will denote an RLD. We also use $n_{i}^{\delta}, P_{k, i}^{\delta}$ and $\kappa_{k, i, t}^{\delta}$ (respectively $n_{i}^{\tau}, P_{k, i}^{\tau}$ and $\kappa_{k, i, t}^{\tau}$ ) for the parameters representing an SLD (respectively an RLD) when it is necessary to make a clear distinction between scheduled and random demands.

\subsection{Bifurcated routing}

Our first routing algorithm we called RWABIF [6] allows traffic bifurcation among several paths between the source and the destination of each lightpath demand. We computed $K$ alternate shortest paths connecting the source to the destination of each demand.

2.2.1 Routing and wavelength assignment of the SLDs. Given a set of SLDs, we want to determine a routing and a wavelength assignment that minimizes the rejection ratio. We define the following additional notations:

- $\Delta=\left\{\delta_{1}, \delta_{2}, \ldots, \delta_{M}\right\}$ is the set of SLDs to be established.

- $(G, \Delta)$ is a pair representing an instance of the SLD routing problem.

- a vector $\left(\rho_{1,1}, \rho_{2,1}, \ldots, \rho_{K, 1}\right)$ is associated to the demand $\delta_{i}$. The element $\rho_{k, i}$ indicates the number of lightpaths to be routed along $P_{k, i}^{\delta}$, the $k^{\text {th }}$ alternate shortest route for SLD $\delta_{i}$.

- $\rho_{\Delta}=\left(\left(\rho_{1,1}, \rho_{2,1}, \ldots, \rho_{K, 1}\right),\left(\rho_{1,2}, \rho_{2,2}, \ldots, \rho_{K, 2}\right), \ldots,\left(\rho_{1, M}, \rho_{2, M}, \ldots\right.\right.$ $\left.\left.\rho_{K, M}\right)\right)$ is called an admissible routing solution for $\Delta$ if $\sum_{k=1}^{K} \rho_{k, i}=$ $n_{i}^{\delta}, 1 \leq i \leq M$.

- $\pi_{\Delta}$ is the set of all admissible routing solutions for $\Delta$.

- $\mathcal{C}: \pi_{\Delta} \rightarrow \mathbb{N}$ is the function that counts the number of blocked SLDs for an admissible solution. The combinatorial optimization problem to solve is:

$$
\begin{array}{cc}
\text { Minimize } & \mathcal{C}\left(\rho_{\Delta}\right) \\
\text { subject to } & \rho_{\Delta} \in \pi_{\Delta}
\end{array}
$$

More details and examples explaining the mathematical model and the way the RWA for the SLDs is computed can be found in [6].

We used a Random Search (RS) algorithm to find an approximate minimum of the function $\mathcal{C}$. The wavelengths are assigned according to a First-Fit scheme.

2.2.2 Routing and wavelength assignment of the RLDs. Once the RWA for the SLDs has been established, we deal with the RLDs sequentially, that is demand by demand at arrival dates. When a new RLD arrives, one tries to route all the requested lightpaths on the shortest path, if it is possible (i.e. if 
there are as many available path-free wavelengths along the shortest path as the requested number of lightpaths), otherwise, several paths between the source and the destination of the demand are used whenever the cumulated number of path-free wavelengths along the considered shortest paths is at least equal to the number of the requested lightpaths, otherwise, the demand is rejected. As they require fewer WDM channels, the path-free wavelengths with shorter paths are preferred to those with longer ones. More details can be found in [6].

\subsection{Non-bifurcated routing}

Our second routing algorithm called RWANBIF imposes non-bifurcated routing: all the requested lightpaths by a LD have to follow the same path between the source node and the destination node of the demand. Traffic splitting is thus prohibited. Again, we computed $K$ alternate shortest paths between the source and the destination of each demand. For each lightpath demand, we try to route the requested number of lightpaths along one of the $K$ shortest paths if this is possible otherwise the LD is rejected.

\subsubsection{Non-bifurcated routing and wavelength assignment of the SLDs.}

Given a set of SLDs, we want to determine a routing and a wavelength assignment that minimize the rejection ratio taking into account the fact that for each SLD, all requested lightpaths have to be routed on a same path between the source and the destination of the demand. We use the same notations as in subsection 2.2.1. Note that this time $\rho_{\Delta}=\left(\left(\rho_{1,1}, \rho_{2,1}, \ldots, \rho_{K, 1}\right),\left(\rho_{1,2}\right.\right.$, $\left.\left.\rho_{2,2}, \ldots, \rho_{K, 2}\right), \ldots,\left(\rho_{1, M}, \rho_{2, M}, \ldots, \rho_{K, M}\right)\right)$ is called an admissible routing solution for $\Delta$ if for each SLD $i(1 \leq i \leq M)$, there exists a unique $j$, $1 \leq j \leq K$ such that $\rho_{j, i}=n_{i}^{\delta}$ and $\rho_{k, i}=0$ for each path $k,(1 \leq k \leq K), k$ different from $j$.

Again, we use a Random Search (RS) algorithm to find an approximate minimum of the function $\mathcal{C}$.

\subsubsection{Non-bifurcated routing and wavelength assignment of the RLDs.}

Once the RWA for the SLDs have been calculated, we establish the RLDs sequentially. All the lightpaths of an RLD are routed through the same path as opposed to the algorithm of subsection 2.2.2. Whenever there are enough available wavelengths on two distinct paths, the shortest one is preferred as it will use less WDM channels.

\section{EXPERIMENTAL RESULTS}

In this section we experimentally evaluate the algorithms proposed in the previous sections. We used two network topologies: the former is the 14node NSFNET network, the latter is the hypothetical US backbone network of 
29 nodes and 44 spans. Due to space limitation we present only the results obtained with the 29-node network. For the sets $\Delta$, the source/destination nodes are drawn according to a random uniform distribution in the interval $[1,14]$ (respectively $[1,29]$ ) for the 14-node network (respectively the 29-node network). We also used uniform random distributions over the intervals $[1,5]$ and $[1,1440]$ for the number of lightpaths and the set-up/tear-down dates of the SLDs respectively. We assume observation periods of about a day (1440 is the number of minutes in a day). Random connection requests (RLDs) arrive according to a Poisson process with an arrival rate $\nu=1$ and if accepted, will hold the circuit for exponentially distributed times with mean $\mu=250$ much larger than the cumulated round-trip time and the connection set-up delay. The number of lightpaths required by an RLD is drawn from a random uniform distribution in the interval $[1,5]$. We call a scenario the set of demands be they scheduled or random that occur from start to finish of a day. We assume that we compute $K=5$ alternate shortest paths between each source/destination pair and that there are $\chi=24$ available wavelengths on each fiber-link in the network. We want to assess the gain obtained using the RWABIF algorithm compared to the RWANBIF algorithm.

We generated 25 test scenarios, run the two algorithms on them and computed rejection ratio averages for each algorithm. Figure 1 shows the average number of rejected SLDs and RLDs when $D$, the total number of lightpath requests arrived at the network, varies from 50 to 500 . Each couple of bars shows the average number of blocked demands computed using the RWABIF (left bar) and the RWANBIF (right bar) algorithms respectively. Each bar is divided into two segments. The height of the black segment indicates the average number of rejected SLDs whereas the height of the white one shows the number of rejected RLDs.

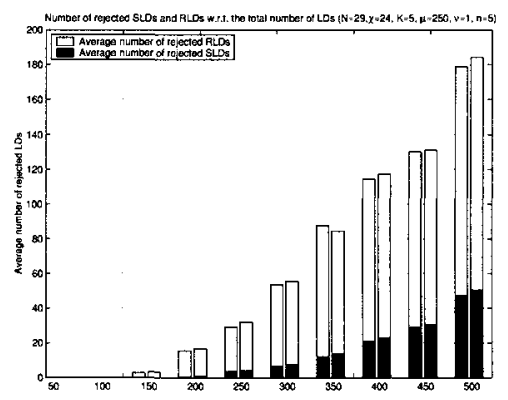

Figure 1. average number of rejected SLDs and RLDs w.r.t. the total number of LDs arrived at the network

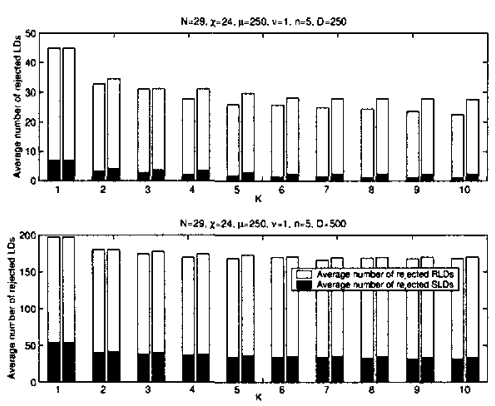

Figure 2. average number of rejected SLDs and RLDs w.r.t. $K$ 
We notice that the global rejection ratio (total number of blocked SLDs and RLDs) increases when $D$ increases. The figure also shows that the RWABIF algorithm computes a lower global rejection ratio than the global rejection ratio computed by the RWANBIF algorithm. A global rejection gain of $5 \%$ is observed. Multipath routing provides better usage of network resources. Note that the number of rejected SLDs computed by the RS algorithm is higher in the case when the traffic is not bifurcated.

In the following figures, we present simulation results obtained with two different values of $D$ (250 and 500$)$. Each figure shows two subfigures. The upper (respectively lower) subfigure shows results obtained when $D=250$ (respectively $D=500$ ).

Figure 2 shows the average number of rejected SLDs and RLDs when $K$, the number of alternate shortest paths, varies from 1 to 10 . We notice that the average number of blocked demands falls when the value of $K$ increases and becomes roughly constant when $K \geq 5$. Note that the global rejection ratio is always higher in the case when the RWANBIF algorithm is used.
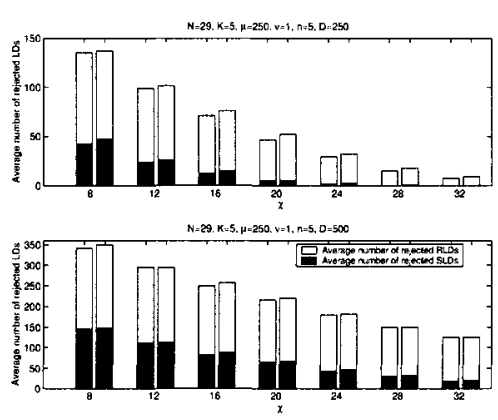

Figure 3. average number of rejected SLDs and RLDs w.r.t. $\chi$
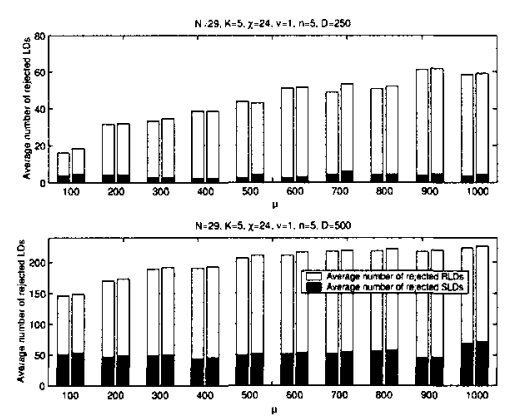

Figure 4. average number of rejected SLDs and RLDs w.r.t. $\mu$

Figure 3 shows the average number of rejected LDs when $\chi$, the number of available wavelengths on each fiber-link of the network, varies from 8 to 32. We notice that the average number of rejected LDs falls when the the number of available wavelengths goes from 8 to 32 . The RWABIF algorithm still services more lightpath demands than the RWANBIF algorithm.

Figure 4 shows the number of rejected LDs w.r.t. $\mu$, the mean duration of RLDs. As $\mu$ increases, more RLDs are rejected and hence the global rejection ratio increases. This is due to the fact that when the mean duration of RLDs increases, the network resources, when affected, are occupied by the RLDs for longer periods and no sufficient resources are available to service an arriving $R L D$. The average number of rejected SLDs remains roughly constant because SLDs are routed independently from RLDs. 


\section{CONCLUSIONS}

In this paper, we proposed two new routing algorithms to deal with the routing and wavelength assignmemnt of both scheduled and random lightpath demands in an all-optical transport network. Both algorithms are presented and compared through rejection ratio. The algorithms process in two separate steps and first compute the RWA for the SLDs and then consider the RLDs on the fly. The RWABIF algorithm allows bifurcated routing as opposed to the RWANBIF algorithm which imposes that all the requested lightpaths of a lightpath demand have to be routed on the same path. We computed the average number of rejected SLDs and RLDs for different simulation scenarios. The results show that a global rejection gain of $5 \%$ is obtained with the RWABIF algorithm at the cost of a significant complexity increase resulting from signalisation messages necessary to the establishment of the various paths in the case when bifurcated routing is allowed.

Future work will focus on how decreasing the rejection ratio. Wavelength rerouting techniques may alleviate the wavelength continuity constraint imposed by the all-optical cross-connect switches.

\section{REFERENCES}

[1] Zang and J. P. Jue and B. Mukherjee, "A Review of Routing and Wavelength Assignment Approaches for Wavelength-Routed Optical WDM Networks", Optical Networks Magazine, vol. 1, no. 1, pp. 47-60, Jan., 2000.

[2] D. Banerjee and B. Mukherjee, "A Practical Aprroach for Routing and Wavelength Assignment in Large Wavelength-Routed Optical Networks", IEEE Journal on Selected Areas in Communications, vol. 14, no. 5, pp. 903-908, Oct., 1996.

[3] M. Alanyali and E. Ayanoglu, "Provisioning Algorithms for WDM Optical Networks", IEEE/ACM Transanctions on Networking, vol. 7, no. 5, pp. 767-778, Oct., 1999.

[4] R. Ramamurthy and B. Mukherjee, "Fixed-Alternate Routing and Wavelength Conversion in Wavelength-Routed Optical Networks", IEEE/ACM Transactions on Networking, vol. 10, no. 3, pp. 351-367, 2002.

[5] R. Ramaswami and K.N. Sivarajan, "Routing and Wavelength Assignment in All-Optical Networks", IEEE/ACM Transactions on Networking, vol. 3, no. 5, pp. 489-500, Oct., 1995.

[6] M. Koubaa and N. Puech and M. Gagnaire, "Routing and Wavelength Assignment of Scheduled and Random Lightpath Demands", Wireless and Optical Communications Networks, Jun. 7-9, 2004.

[7] J. Kuri and N. Puech and M. Gagnaire and E. Dotaro and R. Douville, "Routing and Wavelength Assignment of Scheduled Lightpath Demands", IEEE JSAC Optical Communications and Networking Series, vol. 21, no. 8, pp. 1231-1240, Oct. 2003.

[8] I. Chlamtac and A. Ganz and G. Karmi, "Purely Optical Networks for Terabit Communication", Proc. IEEE INFOCOM, pp. 887-896, 1989.

[9] D. Eppstein, "Finding the $k$ Shortest Paths", SIAM Journal of Computing, vol. 28, no. 2, pp. 652-673, 1998. 